\title{
Boundary Value Problems of Fractional Order Differential Equation with Integral Boundary Conditions and Not Instantaneous Impulses
}

\author{
Peiluan $\mathrm{Li}^{1}$ and Changjin $\mathrm{Xu}^{2}$ \\ ${ }^{1}$ Department of Mathematics and Statistics, Henan University of Science and Technology, Luoyang 471023, China \\ ${ }^{2}$ Guizhou Key Laboratory of Economics System Simulation, Guizhou College of Finance and Economics, Guiyang 550004, China \\ Correspondence should be addressed to Peiluan Li; lpllpl_lpl@163.com
}

Received 3 October 2014; Revised 11 February 2015; Accepted 22 February 2015

Academic Editor: Jesús G. Falset

Copyright (C) 2015 P. Li and C. Xu. This is an open access article distributed under the Creative Commons Attribution License, which permits unrestricted use, distribution, and reproduction in any medium, provided the original work is properly cited.

We investigate the existence of mild solutions for fractional order differential equations with integral boundary conditions and not instantaneous impulses. By some fixed-point theorems, we establish sufficient conditions for the existence and uniqueness of solutions. Finally, two interesting examples are given to illustrate our theory results.

\section{Introduction}

Impulsive differential equations are used to describe many practical dynamical systems including evolutionary processes characterized by abrupt changes of the state at certain instants. Such processes are naturally seen in biology, physics, engineering, and so forth. Due to their significance, many authors have established the solvability of impulsive differential equations. Nowadays, the theory of impulsive differential equations has received great attention. Differential equations with instantaneous impulses have been treated in several works (see, e.g., the monographs [1-3], the works on time variable impulses problem [4-7], and the references therein).

However, in almost all the papers concerning impulsive differential equations, the impulses are all instantaneous impulses, and the classical models with instantaneous impulses cannot characterize many practical problems, for example, the dynamics of evolution processes in pharmacotherapy. Let us consider the hemodynamic equilibrium of a person. The introduction of the drugs in the bloodstream and the consequent absorption for the body are gradual and continuous processes. In fact, this situation should be characterized by a new case of impulsive action, which starts at an arbitrary fixed point $t_{i}$ and stays active on a finite time interval $\left[t_{i}, s_{i}\right]$. To this end, Hernández and O’Regan [8] initially offered to study a new class of abstract semilinear impulsive differential equations with not instantaneous impulses in a PC-normed Banach space. In [8], the authors discussed the following problem:

$$
\begin{gathered}
u^{\prime}(t)=A u(t)+f(t, u(t)), \quad t \in\left(s_{i}, t_{i+1}\right], i=0, \ldots, N, \\
u(t)=g_{i}(t, u(t)), \quad t \in\left(t_{i}, s_{i}\right], i=1, \ldots, N, \\
u(0)=x_{0},
\end{gathered}
$$

where $A: D(A) \subset X \rightarrow X$ is the generator of a $C_{0}$ semigroup of bounded linear operators $(T(t))_{t>0}$ defined on a Banach space $(X,\|\cdot\|), 0=t_{0}=s_{0}<t_{1} \leq s_{1} \leq \cdots \leq t_{N} \leq s_{N} \leq$ $t_{N+1}=a$ are prefixed numbers, $x_{0} \in X, g_{i} \in C\left(\left(t_{i}, s_{i}\right] \times X, X\right)$ for all $i=1, \ldots, N$, and $f:[0, a] \times X \rightarrow X$ is a suitable function. Meanwhile, Pierri et al. [9] continued the work in [8] in a $\mathrm{PC}_{\alpha}$-normed Banach space.

On the one hand, the absorption of drugs has a memory effect; thus, the new class of impulsive conditions introduced by [8] may not explain this phenomenon very well. On the other hand, fractional calculus provides a powerful tool for the description of hereditary properties of various materials and memory processes $[10,11]$. Fractional differential equations have recently proved to be strong tools in the modeling 
of medical, physics, economics, and technical sciences. For more details on fractional calculus theory, one can see the monographs of Diethelm [12], Kilbas et al. [13], Lakshmikantham et al. [14], Miller and Ross [15], Podlubny [16], and Tarasov [17]. Fractional differential equations involving the Riemann-Liouville fractional derivative or the Caputo fractional derivative have been paid more and more attention (see $[10,11,18-22])$.

The theory of boundary value problems (BVPs) with integral boundary conditions for differential equations arises in different areas of applied mathematics and physics. For example, heat conduction, chemical engineering, underground water flow, thermo-elasticity, and plasma physics can be reduced to the nonlocal problems with integral boundary conditions. For BVPs with integral boundary conditions and comments on their importance, we refer the readers to the papers by Gallardo [23], Karakostas and Tsamatos [24], Lomtatidze and Malaguti [25], and the references therein. For more information about the general theory of integral equations and their relation with BVPs, we refer to the books of Corduneanu [26] and Agarwal and O'Regan [27]. Moreover, BVPs with integral boundary conditions constitute a very interesting and important class of problems. They include two, three, multipoint, and nonlocal BVPs as special cases. The existence and multiplicity of positive solutions for such problems have received a great deal of attention. To identify a few, we refer the readers to [28-31] and references therein.

In [32], the authors consider the following problem:

$$
\begin{gathered}
{ }^{c} D_{t}^{q} u(t)=f\left(t, u(t), \int_{0}^{t} k(t, s, u(s)) d s\right), \\
t \in J^{\prime}:=J \backslash\left\{t_{1}, t_{2}, \ldots, t_{m}\right\}, \quad J:=[0, T], \\
u\left(t_{k}^{+}\right)=u\left(t_{k}^{-}\right)+u_{k}, \quad u_{k} \in R, k=0,1, \ldots, m, \\
u(0)=\int_{0}^{1} g(s) u(s) d s,
\end{gathered}
$$

where ${ }^{c} D_{t}^{q}$ is the Caputo fractional derivative of order $q \epsilon$ $(0,1)$ with the lower limit zero, $f: J \times X \times X \rightarrow X$ is a given function, $k: \Delta \times X \rightarrow X, g \in L^{1}\left([0,1], R^{+}\right)$, $g(t) \in[0,1), \Delta=\{(t, s): 0 \leq s \leq t \leq 1\}$, and $t_{k}$ satisfy $0=$ $t_{0}<t_{1}<\cdots<t_{m+1}=T, u\left(t_{k}^{+}\right)=\lim _{h \rightarrow 0^{+}} u\left(t_{k}+h\right)$ and $u\left(t_{k}^{-}\right)=\lim _{h \rightarrow 0^{-}} u\left(t_{k}+h\right)$ represent the right and left limits of $u(t)$ at $t=t_{k}$. Obviously, the impulses in (2) are instantaneous. Motivated by the work in $[8,9,32]$, in this paper, we consider the following impulsive fractional differential equations with integral boundary conditions and not instantaneous impulses:

$$
\begin{gathered}
{ }^{c} D_{t}^{q} u(t)=f\left(t, u(t), \int_{0}^{t} k(t, s, u(s)) d s\right), \\
t \in\left(s_{i}, t_{i+1}\right], \quad i=0, \ldots, N, \\
u(t)=g_{i}(t, u(t)), \quad t \in\left(t_{i}, s_{i}\right], i=1, \ldots, N, \\
u(0)=\int_{0}^{T} w(s) u(s) d s,
\end{gathered}
$$

where ${ }^{c} D_{t}^{q}$ is the Caputo fractional derivative of order $q \epsilon$ $(0,1)$ with the lower limit zero, $0=t_{0}=s_{0}<t_{1} \leq s_{1} \leq \cdots \leq$ $t_{N} \leq s_{N} \leq t_{N+1}=T$ are prefixed numbers, $g_{i} \in C\left(\left(t_{i}, s_{i}\right] \times\right.$ $R, R)$, for $i=1, \ldots, N, J=[0, T], f: J \times R \times R \rightarrow R$, $k: \Delta \times R \rightarrow R, w \in L^{1}\left([0, T], R^{+}\right)$, and $w \in[0,1 / T), \Delta=$ $\{(t, s): 0 \leq s \leq t \leq T\}$.

The rest of this paper is organized as follows. In Section 2, some lemmas which are essential to prove our main results are stated. In Section 3, we give the main results. In Section 4, two interesting examples are given to illustrate our theory results.

\section{Preliminaries}

At first, we present the necessary definitions for the fractional calculus theory.

Definition 1 (see [13]). The Riemann-Liouville fractional integral of order $\alpha>0$ of a suitable function $y:(0, \infty) \rightarrow R$ is given by

$$
I_{0_{+}}^{\alpha} y(t)=\frac{1}{\Gamma(\alpha)} \int_{0}^{t}(t-s)^{\alpha-1} y(s) d s,
$$

where the right side is pointwise defined on $(0,+\infty)$.

Definition 2 (see [13]). The Caputo fractional derivative of order $\alpha>0$ of a suitable function $y:(0, \infty) \rightarrow R$ is given by

$$
{ }^{c} D^{\alpha} y(t)=\frac{1}{\Gamma(n-\alpha)} \int_{0}^{t}(t-s)^{n-\alpha-1} y^{(n)}(s) d s,
$$

where $n=[\alpha]+1,[\alpha]$ denotes the integer part of number $\alpha$ and the right side is pointwise defined on $(0,+\infty)$.

Lemma 3 (see [13]). Let $\alpha>0$; then the fractional differential equation ${ }^{c} D^{\alpha} u(t)=0$ has solutions

$$
u(t)=c_{0}+c_{1} t+c_{2} t^{2}+\cdots+c_{n-1} t^{n-1},
$$

where $c_{i} \in R, i=0,1, \ldots, n-1, n=[\alpha]+1$.

Lemma 4 (see [13]). Let $\alpha>0$, then one has

$$
I_{0_{+}}^{\alpha c} D^{\alpha} u(t)=u(t)+c_{0}+c_{1} t+c_{2} t^{2}+\cdots+c_{n-1} t^{n-1},
$$

where $c_{i} \in R, i=0,1, \ldots, n-1, n=[\alpha]+1$.

Lemma 5 (Krasnoselskii's fixed point theorem [33]). Let $M$ be a closed convex and nonempty subset of a Banach space $X$. Let $A$ and $B$ be two operators such that

(1) $A x+B y \in M$ whenever $x, y \in M$;

(2) $A$ is compact and continuous;

(3) B is a contraction mapping.

Then there exists $z \in M$ such that $z=A z+B z$.

In order to study problem (3), we define $X=P C(J, R)=$ $\left\{x: J \rightarrow R ; x \in C\left(\left(t_{k}, t_{k+1}\right], R\right), k=0,1, \ldots, N\right.$, and $x\left(t_{k}^{+}\right)$, $x\left(t_{k}^{-}\right)$exist with $\left.x\left(t_{k}^{-}\right)=x\left(t_{k}\right), k=1, \ldots, N\right\}$.

It is easy to check that $X$ is a Banach space with the norm $\|x\|_{P C}=\sup _{t \in J}|x(t)|$. 
Let $B u(t)=\int_{0}^{t} k(t, s, u(s)) d s, \sigma=\int_{0}^{t_{1}} w(t) d t$; then $0 \leq \sigma \leq$ $\int_{0}^{T} w(t) d t<1$.

If $u \in \operatorname{PC}(J, R)$ satisfies problem (3), then for $t \in\left(s_{i}, t_{i+1}\right]$, $i=1, \ldots, N$, integrating the first equation of (3) from $s_{i}$ to $t$ by virtue of Definition 1, one can obtain

$$
u(t)=u\left(s_{i}\right)+\frac{1}{\Gamma(q)} \int_{s_{i}}^{t}(t-s)^{q-1} f(s, u(s), B u(s)) d s .
$$

From the second equation in (3), we know $u\left(s_{i}\right)=g_{i}\left(s_{i}, u\left(s_{i}\right)\right)$. Then, for $t \in\left(s_{i}, t_{i+1}\right]$, we have

$u(t)$

$$
=g_{i}\left(s_{i}, u\left(s_{i}\right)\right)+\frac{1}{\Gamma(q)} \int_{s_{i}}^{t}(t-s)^{q-1} f(s, u(s), B u(s)) d s .
$$

For $t \in\left[0, t_{1}\right]$, integrating the first equation in (3) from 0 to $t$ by virtue of Definition 1 , one can obtain

$$
u(t)=u(0)+\frac{1}{\Gamma(q)} \int_{0}^{t}(t-s)^{q-1} f(s, u(s), B u(s)) d s .
$$

By the boundary conditions, we have

$$
\begin{aligned}
u(t)= & \int_{0}^{T} w(t) u(t) d t \\
& +\frac{1}{\Gamma(q)} \int_{0}^{t}(t-s)^{q-1} f(s, u(s), B u(s)) d s .
\end{aligned}
$$

Multiplying (11) with $w(t)$ and integrating from 0 to $t_{1}$, we have

$$
\begin{aligned}
& \int_{0}^{t_{1}} w(t) u(t) d t \\
& \quad=\int_{0}^{t_{1}} w(t) d t \int_{0}^{T} w(s) u(s) d s \\
& \quad+\frac{1}{\Gamma(q)} \int_{0}^{t_{1}} w(t) \int_{0}^{t}(t-s)^{q-1} f(s, u(s), B u(s)) d s d t,
\end{aligned}
$$

Multiplying (9) with $w(t)$ and integrating from $s_{i}$ to $t_{i+1}, i=$ $1, \ldots, N$, we have

$$
\begin{aligned}
& \int_{s_{i}}^{t_{i+1}} w(t) u(t) d t \\
&= g_{i}\left(s_{i}, u\left(s_{i}\right)\right) \int_{s_{i}}^{t_{i+1}} w(t) d t+\frac{1}{\Gamma(q)} \\
& \quad \cdot \int_{s_{i}}^{t_{i+1}} w(t) \frac{1}{\Gamma(q)} \int_{s_{i}}^{t}(t-s)^{q-1} f(s, u(s), B u(s)) d s d t .
\end{aligned}
$$

Multiplying the second equation of (3) with $w(t)$ and integrating from $t_{i}$ to $s_{i}, i=1, \ldots, N$, we can obtain

$$
\int_{t_{i}}^{s_{i}} w(t) u(t) d t=\int_{t_{i}}^{s_{i}} w(t) g_{i}(t, u(t)) d t .
$$

Adding (12), (13), and (14), one has

$$
\begin{aligned}
& \int_{0}^{T} w(t) u(t) d t \\
& =\int_{0}^{t_{1}} w(t) d t \int_{0}^{T} w(s) u(s) d s+\sum_{i=1}^{N} g_{i}\left(s_{i}, u\left(s_{i}\right)\right) \int_{s_{i}}^{t_{i+1}} w(t) d t \\
& \quad+\frac{1}{\Gamma(q)} \sum_{i=0}^{N} \int_{s_{i}}^{t_{i+1}} w(t) \int_{s_{i}}^{t}(t-s)^{q-1} f(s, u(s), B u(s)) d s d t \\
& \quad+\sum_{i=1}^{N} \int_{t_{i}}^{s_{i}} w(t) g_{i}(t, u(t)) d t .
\end{aligned}
$$

Hence

$$
\begin{aligned}
& \int_{0}^{T} w(t) u(t) d t \\
& =\frac{1}{1-\sigma} \\
& \quad \cdot\left\{\sum_{i=1}^{N} g_{i}\left(s_{i}, u\left(s_{i}\right)\right) \int_{s_{i}}^{t_{i+1}} w(t) d t\right. \\
& \quad+\frac{1}{\Gamma(q)} \sum_{i=0}^{N} \int_{s_{i}}^{t_{i+1}} w(t) \int_{s_{i}}^{t}(t-s)^{q-1} f(s, u(s), B u(s)) d s d t \\
& \left.\quad+\sum_{i=1}^{N} \int_{t_{i}}^{s_{i}} w(t) g_{i}(t, u(t)) d t\right\} .
\end{aligned}
$$

So, for $t \in\left[0, t_{1}\right]$, we have

$$
\begin{aligned}
& u(t) \\
& =\frac{1}{\Gamma(q)} \int_{0}^{t}(t-s)^{q-1} f(s, u(s), B u(s)) d s \\
& +\frac{1}{1-\sigma} \\
& \quad \cdot\left\{\sum_{i=1}^{N} g_{i}\left(s_{i}, u\left(s_{i}\right)\right) \int_{s_{i}}^{t_{i+1}} w(t) d t+\frac{1}{\Gamma(q)}\right. \\
& \quad \cdot \sum_{i=0}^{N} \int_{s_{i}}^{t_{i+1}} w(t) \int_{s_{i}}^{t}(t-s)^{q-1} f(s, u(s), B u(s)) d s d t \\
& \left.\quad+\sum_{i=1}^{N} \int_{t_{i}}^{s_{i}} w(t) g_{i}(t, u(t)) d t\right\} .
\end{aligned}
$$

Then similar to Definition 2.1 in [9], we can define the mild solution for (3). 
Definition 6. A function $u \in \mathrm{PC}(J, R)$ is a mild solution of problems (3) if

$$
\begin{gathered}
u(0)=\int_{0}^{T} w(s) u(s) d s, \\
u(t)=g_{i}(t, u(t)), \quad \forall t \in\left(t_{i}, s_{i}\right], i=1, \ldots, N,
\end{gathered}
$$

$u(t)$

$$
\begin{aligned}
= & \frac{1}{\Gamma(q)} \int_{0}^{t}(t-s)^{q-1} f(s, u(s), B u(s)) d s+\frac{1}{1-\sigma} \\
& \cdot\left\{\sum_{i=1}^{N} g_{i}\left(s_{i}, u\left(s_{i}\right)\right) \int_{s_{i}}^{t_{i+1}} w(t) d t+\frac{1}{\Gamma(q)}\right. \\
& \cdot \sum_{i=0}^{N} \int_{s_{i}}^{t_{i+1}} w(t) \int_{s_{i}}^{t}(t-s)^{q-1} f(s, u(s), B u(s)) d s d t \\
& \left.+\sum_{i=1}^{N} \int_{t_{i}}^{s_{i}} w(t) g_{i}(t, u(t)) d t\right\}
\end{aligned}
$$

for all $t \in\left[0, t_{1}\right]$ and

$$
\begin{aligned}
& u(t) \\
& \quad=g_{i}\left(s_{i}, u\left(s_{i}\right)\right)+\frac{1}{\Gamma(q)} \int_{s_{i}}^{t}(t-s)^{q-1} f(s, u(s), B u(s)) d s
\end{aligned}
$$

for all $t \in\left(s_{i}, t_{i+1}\right], i=1, \ldots, N$

\section{Main Results}

This section deals with the existence of mild solutions for (3). Before stating and proving the main results, we make the following hypotheses.

$\left(H_{1}\right) f: J \times R \times R \rightarrow R$ is jointly continuous. There exists a function $e \in C(J, R)$ such that

$$
\begin{aligned}
& \left|f\left(t, u_{1}(t), v_{1}(t)\right)-f\left(t, u_{2}(t), v_{2}(t)\right)\right| \\
& \quad \leq e(t)\left(\left|u_{1}(t)-u_{2}(t)\right|+\left|v_{1}(t)-v_{2}(t)\right|\right),
\end{aligned}
$$

for all $u_{1}, v_{1}, u_{2}, v_{2} \in \operatorname{PC}(J, R)$, for all $t \in J$.

$\left(H_{2}\right) k: \Delta \times R \rightarrow R$ is continuous and there exists a function $d \in C\left[J, R^{+}\right]$such that

$$
\begin{array}{r}
|k(t, s, u(s))-k(t, s, v(s))| \leq d(t)(|u(t)-v(t)|), \\
\forall u, v \in \operatorname{PC}(J, R), \quad(t, s) \in \Delta .
\end{array}
$$

$\left(H_{3}\right) g_{i} \in C\left(\left(t_{i}, s_{i}\right] \times R, R\right)$ and there exist $l_{i} \in C[J, R], i=$ $1, \ldots, N$ such that

$$
\begin{array}{r}
\left|g_{i}(t, x(t))-g_{i}(t, y(t))\right| \leq l_{i}(t)|x(t)-y(t)|, \\
\forall x, y \in \mathrm{PC}(J, R), \quad \forall t \in J .
\end{array}
$$

Let

$$
\begin{gathered}
L=\max _{1 \leq i \leq N} \sup _{t \in J}\left|l_{i}(t)\right|, \quad M=\sup _{t \in J}|e(t)|, \\
D=\sup _{t \in J}|d(t)| .
\end{gathered}
$$

Now we are in the position to establish the main results. Our first theorem is based on contraction mapping principle.

Theorem 7. Let $\left(H_{1}\right)-\left(H_{3}\right)$ hold and $n<1$; then the problem (3) has a unique mild solution, where

$$
n=\frac{T^{q} M(1+T D)(2-\sigma)}{\Gamma(q+1)(1-\sigma)}+\frac{2 L}{1-\sigma} .
$$

Proof. Let $A: \mathrm{PC}(J, R) \rightarrow \mathrm{PC}(J, R)$ be the map defined by

$$
A u(t)=g_{i}(t, u(t)), \quad \text { for } t \in\left(t_{i}, s_{i}\right], i=1, \ldots, N,
$$

$A u(t)$

$$
\begin{aligned}
& =\frac{1}{\Gamma(q)} \int_{0}^{t}(t-s)^{q-1} f(s, u(s), B u(s)) d s+\frac{1}{1-\sigma} \\
& \quad \cdot\left\{\sum_{i=1}^{N} g_{i}\left(s_{i}, u\left(s_{i}\right)\right) \int_{s_{i}}^{t_{i+1}} w(t) d t+\frac{1}{\Gamma(q)}\right. \\
& \quad \cdot \sum_{i=0}^{N} \int_{s_{i}}^{t_{i+1}} w(t) \int_{s_{i}}^{t}(t-s)^{q-1} f(s, u(s), B u(s)) d s d t \\
& \left.\quad+\sum_{i=1}^{N} \int_{t_{i}}^{s_{i}} w(t) g_{i}(t, u(t)) d t\right\}
\end{aligned}
$$

for $t \in\left[0, t_{1}\right]$ and

$$
\begin{aligned}
A u(t)= & g_{i}\left(s_{i}, u\left(s_{i}\right)\right) \\
& +\frac{1}{\Gamma(q)} \int_{s_{i}}^{t}(t-s)^{q-1} f(s, u(s), B u(s)) d s
\end{aligned}
$$

for $t \in\left(s_{i}, t_{i+1}\right], i=1, \ldots, N$. Clearly, $A$ is well defined.

Next we show that $A$ is contraction on $\operatorname{PC}(J, R)$.

Fix $x, y \in \mathrm{PC}(J, R)$; we consider three cases.

Case 1. If $t \in\left(s_{i}, t_{i+1}\right], i=1, \ldots, N$, by the assumptions $\left(H_{1}\right)-\left(H_{3}\right)$ and the property $\Gamma(q+1)=q \Gamma(q)$, we have

$$
\begin{aligned}
& |A x(t)-A y(t)| \\
& \leq\left|g_{i}\left(s_{i}, x\left(s_{i}\right)\right)-g_{i}\left(s_{i}, y\left(s_{i}\right)\right)\right| \\
& +\frac{1}{\Gamma(q)} \int_{0}^{t}(t-s)^{q-1} \\
& \quad \cdot|f(s, x(s), B x(s)) d s-f(s, y(s), B y(s))| d s
\end{aligned}
$$




$$
\begin{aligned}
\leq & L\|x-y\|_{\mathrm{PC}}+\left.\frac{M}{\Gamma(q)}\left[-\frac{(t-s)^{q}}{q}\right]\right|_{0} ^{t} \\
& \cdot\left(\|x-y\|_{\mathrm{PC}}+\|B x-B y\|_{\mathrm{PC}}\right) \\
= & L\|x-y\|_{\mathrm{PC}}+\frac{M}{\Gamma(q)} \frac{t^{q}}{q}\left(\|x-y\|_{\mathrm{PC}}+\|B x-B y\|_{\mathrm{PC}}\right) \\
\leq & L\|x-y\|_{\mathrm{PC}}+\frac{T^{q} M}{\Gamma(q+1)}\left(\|x-y\|_{\mathrm{PC}}+\|B x-B y\|_{\mathrm{PC}}\right) \\
\leq & {\left[L+\frac{T^{q} M(1+T D)}{\Gamma(q+1)}\right]\|x-y\|_{\mathrm{PC}} . }
\end{aligned}
$$

Case 2. If $t \in\left[0, t_{1}\right]$, by $\left(H_{1}\right),\left(H_{2}\right)$, one can obtain

$$
\begin{aligned}
& |A x(t)-A y(t)| \\
& \leq \frac{1}{\Gamma(q)} \int_{0}^{t}(t-s)^{q-1} \\
& \quad \cdot|f(s, x(s), B x(s))-f(s, y(s), B y(s))| d s \\
& +\frac{1}{1-\sigma}\left\{\sum_{i=1}^{N}\left|g_{i}\left(s_{i}, x\left(s_{i}\right)\right)-g_{i}\left(s_{i}, y\left(s_{i}\right)\right)\right| \int_{s_{i}}^{t_{i+1}} w(t) d t\right. \\
& \quad+\frac{1}{\Gamma(q)} \sum_{i=0}^{N} \int_{s_{i}}^{t_{i+1}} w(t) \int_{s_{i}}^{t}(t-s)^{q-1} \\
& \cdot \mid f(s, x(s), B x(s)) \\
& \left.\quad+\sum_{i=1}^{N} \int_{t_{i}}^{s_{i}} w(t)\left|g_{i}(t, x(t))-g_{i}(t, y(t))\right| d t\right\} .
\end{aligned}
$$

Then, by a similar argument, we can get

$$
\begin{aligned}
& |A x(t)-A y(t)| \\
& \quad \leq\left(\frac{T^{q} M(1+T D)(2-\sigma)}{\Gamma(q+1)(1-\sigma)}+\frac{2 L}{1-\sigma}\right)\|x-y\|_{\mathrm{PC}} .
\end{aligned}
$$

Case 3. If $t \in\left(t_{i}, s_{i}\right], i=1, \ldots, N$, from the assumption $\left(H_{3}\right)$, we get

$$
|A x(t)-A y(t)| \leq L\|x-y\|_{\mathrm{PC}} .
$$

Therefore, $\|A x-A y\|_{\mathrm{PC}} \leq n\|x-y\|_{\mathrm{PC}}$, for all $x, y \in$ $\mathrm{PC}(J, R)$, which implies that $A$ is a contraction mapping. Then, there exists a unique mild solution of (3). $\left(H_{4}\right)$.

In order to get the second main result, we give assumption

$\left(H_{4}\right)$ The function $f: J \times R \times R \rightarrow R$ is jointly continuous and strongly measurable on $J$. There exist $m_{f} \in C\left(J, R^{+}\right)$and a nondecreasing function $h_{f} \in$ $C\left(R^{+}, R^{+}\right)$such that

$$
|f(t, x, y)| \leq m_{f}(t) h_{f}(|x+y|) \quad \forall t \in J, x, y \in R .
$$

Our second result is based on Krasnoselskii's fixed point theorem.

Theorem 8. Assume that $\left(H_{2}\right)-\left(H_{4}\right)$ hold; if $2 L /(1-\sigma)<1$ and there exists a constant $r>0$ such that

$$
\begin{aligned}
& r(1-\sigma-2 L) \\
& \quad \geq \frac{T^{q}(2-\sigma)}{\Gamma(q+1)}\left\|m_{f}\right\|_{P C} h_{f}[r(1+T D)+T K]+2 a,
\end{aligned}
$$

where $a=\max _{i=1, \ldots, N}\left|g_{i}(t, 0)\right|$, then the problem (3) has at least a mild solution.

Proof. Let $A u(t)$ be the map introduced in the proof of Theorem 7. We consider the decomposition $A u(t)=A^{1} u(t)+$ $A^{2} u(t)$, where

$$
\begin{aligned}
& A^{1} u(t)=\sum_{i=1}^{N} A_{i}^{1} u(t), \quad A^{2} u(t)=\sum_{i=1}^{N} A_{i}^{2} u(t), \\
& A_{i}^{1} u(t)=\left\{\begin{array}{c}
\frac{1}{\Gamma(q)} \int_{s_{i}}^{t}(t-s)^{q-1} f(s, u(s), B u(s)) d s, \\
\text { if } t \in\left(s_{i}, t_{i+1}\right], \quad i \geq 1, \\
\frac{1}{\Gamma(q)} \int_{0}^{t}(t-s)^{q-1} f(s, u(s), B u(s)) d s \\
+\frac{1}{(1-\sigma) \Gamma(q)} \\
\cdot \sum_{i=0}^{N} \int_{s_{i}}^{t_{i+1}} w(t) \int_{s_{i}}^{t}(t-s)^{q-1} \\
\cdot f(s, u(s), B u(s)) d s d t,
\end{array}\right. \\
& \text { if } t \in\left[0, t_{1}\right] \text {, } \\
& 0 \text {, else, } \\
& A_{i}^{2} u(t)=\left\{\begin{array}{cc}
g_{i}(t, u(t)), & \text { if } t \in\left(t_{i}, s_{i}\right], i \geq 1, \\
g_{i}\left(s_{i}, u\left(s_{i}\right)\right), & \text { if } t \in\left(s_{i}, t_{i+1}\right], i \geq 1, \\
\frac{1}{1-\sigma}\left\{\sum_{i=1}^{N} g_{i}\left(s_{i}, u\left(s_{i}\right)\right) \int_{s_{i}}^{t_{i+1}} w(t) d t\right. \\
\left.+\sum_{i=1}^{N} \int_{t_{i}}^{s_{i}} w(t) g_{i}(t, u(t)) d t\right\} \\
\text { if } t \in\left[0, t_{1}\right], \\
\text { else. }
\end{array}\right.
\end{aligned}
$$

Let $B_{r}=\left\{x \in \mathrm{PC}(J, R):\|x\|_{\mathrm{PC}} \leq r\right\}$. We divide our proof into three steps. 
Step 1. First we show that $A^{1} x+A^{2} y \in B_{r}$ whenever $x, y \in B_{r}$.

From $\left(H_{2}\right)$, we know that $k: \Delta \times X \rightarrow X$ is continuous, and then $k(t, s, 0)$ is bounded, for $(t, s) \in \Delta=\{(t, s): 0 \leq s \leq$ $t \leq T\}$. Let $K:=\max \{k(t, s, 0):(t, s) \in \Delta\}$.

Let $x \in B_{r}$; if $t \in\left(s_{i}, t_{i+1}\right]$, we have

$$
\begin{aligned}
& \left|A_{i}^{1} x(t)\right| \\
& \quad \leq \frac{1}{\Gamma(q)} \int_{s_{i}}^{t}(t-s)^{q-1}|f(s, x(s), B x(s))| d s \\
& \quad \leq \frac{1}{\Gamma(q)}\left(\int_{s_{i}}^{t}(t-s)^{q-1} m_{f}(s) d s\right) h_{f}\left(\|x+B x\|_{\mathrm{PC}}\right) \\
& \quad \leq \frac{1}{\Gamma(q)}\left(\int_{s_{i}}^{t}(t-s)^{q-1} d s\right)\left\|m_{f}\right\|_{\mathrm{PC}} h_{f}\left(\|x+B x\|_{\mathrm{PC}}\right) \\
& \quad \leq \frac{1}{\Gamma(q)}\left(\int_{0}^{t}(t-s)^{q-1} d s\right)\left\|m_{f}\right\|_{\mathrm{PC}} h_{f}\left(\|x+B x\|_{\mathrm{PC}}\right) \\
& \quad=\left.\frac{1}{\Gamma(q)}\left[-\frac{(t-s)^{q}}{q}\right]\right|_{0} ^{t}\left\|m_{f}\right\|_{\mathrm{PC}} h_{f}\left(\|x+B x\|_{\mathrm{PC}}\right) \\
& \quad=\frac{1}{\Gamma(q)} \frac{t^{q}}{q}\left\|m_{f}\right\|_{\mathrm{PC}} h_{f}\left(\|x+B x\|_{\mathrm{PC}}\right) \\
& \quad \leq \frac{T^{q}}{q \Gamma(q)}\left\|m_{f}\right\|_{\mathrm{PC}} h_{f}\left(\|x\|_{\mathrm{PC}}+\|B x\|_{\mathrm{PC}}\right) .
\end{aligned}
$$

By the definition of $B$, the integral mean value theorem, $\left(\mathrm{H}_{2}\right)$, and the property $\Gamma(q+1)=q \Gamma(q)$, we have

$$
\begin{aligned}
\left|A_{i}^{1} x(t)\right| & \\
\leq & \frac{T^{q}}{\Gamma(q+1)}\left\|m_{f}\right\|_{\mathrm{PC}} \\
& \cdot h_{f}\left[\|x\|_{\mathrm{PC}}+T(|k(t, \xi, x)-k(t, \xi, 0)|+|k(t, \xi, 0)|)\right] \\
\leq & \frac{T^{q}}{\Gamma(q+1)}\left\|m_{f}\right\|_{\mathrm{PC}} \\
& \cdot h_{f}\left(\|x\|_{\mathrm{PC}}+D T\|x\|_{\mathrm{PC}}+T|k(t, \xi, 0)|\right) \\
\leq & \frac{T^{q}}{\Gamma(q+1)}\left\|m_{f}\right\|_{\mathrm{PC}} h_{f}[r(1+T D)+T K],
\end{aligned}
$$

where $0 \leq \xi \leq t \leq T$.

By a similar argument, let $x \in B_{r}$, if $t \in\left[0, t_{1}\right]$; we have

$$
\begin{aligned}
& \left|A_{i}^{1} x(t)\right| \\
& \leq \frac{1}{\Gamma(q)} \int_{0}^{t}(t-s)^{q-1} f(s, x(s), B x(s)) d s \\
& \quad+\frac{1}{(1-\sigma) \Gamma(q)} \\
& \quad \cdot \sum_{i=0}^{N} \int_{s_{i}}^{t_{i+1}} w(t) \int_{s_{i}}^{t}(t-s)^{q-1} f(s, x(s), B x(s)) d s d t
\end{aligned}
$$

$$
\begin{aligned}
\leq & \frac{T^{q}}{\Gamma(q+1)}\left\|m_{f}\right\|_{\mathrm{PC}} h_{f}[r(1+T D)+T K] \\
& +\frac{1}{(1-\sigma) \Gamma(q)} \\
& \cdot \int_{0}^{T} w(t) \frac{T^{q}}{q}\left\|m_{f}\right\|_{\mathrm{PC}} h_{f}[r(1+T D)+T K] d t \\
= & \frac{T^{q}}{\Gamma(q+1)}\left(1+\frac{\int_{0}^{T} w(t) d t}{1-\sigma}\right)\left\|m_{f}\right\|_{\mathrm{PC}} \\
& \cdot h_{f}[r(1+T D)+T K] .
\end{aligned}
$$

From the condition $w \in L^{1}\left([0, T], R^{+}\right), w \in[0,1 / T)$, we can get

$$
\left|A_{i}^{1} x(t)\right| \leq \frac{T^{q}(2-\sigma)}{\Gamma(q+1)(1-\sigma)}\left\|m_{f}\right\|_{\mathrm{PC}} h_{f}[r(1+T D)+T K] .
$$

For the other cases, from the definition of $A_{i}^{1} u$, one can get $\left|A_{i}^{1} x(t)\right|=0$.

From the proof above, let $x \in B_{r}$; then for all $t \in[0, T]$, we have

$$
\left|A_{i}^{1} x(t)\right| \leq \frac{T^{q}(2-\sigma)}{\Gamma(q+1)(1-\sigma)}\left\|m_{f}\right\|_{\mathrm{PC}} h_{f}[r(1+T D)+T K] .
$$

Let $y \in B_{r}$; if $t \in\left(t_{i}, s_{i}\right], i \geq 1$, we can obtain

$$
\left|A_{i}^{2} y(t)\right| \leq\left|g_{i}(t, y(t))-g_{i}(t, 0)\right|+\left|g_{i}(t, 0)\right| \leq L r+a .
$$

Let $y \in B_{r}$; if $t \in\left[0, t_{1}\right]$, we can get

$$
\begin{gathered}
\left|A_{i}^{2} y(t)\right| \\
\leq \frac{1}{1-\sigma}\left\{\sum _ { i = 1 } ^ { N } \left(\left(\left|g_{i}\left(s_{i}, y\left(s_{i}\right)\right)-g_{i}\left(s_{i}, 0\right)\right|\right.\right.\right. \\
\left.\left.+\left|g_{i}\left(s_{i}, 0\right)\right|\right) \int_{s_{i}}^{t_{i+1}} w(t) d t\right) \\
+\sum_{i=1}^{N} \int_{t_{i}}^{s_{i}} w(t)\left(\left|g_{i}(t, y(t))-g_{i}(t, 0)\right|\right. \\
\left.\left.+\left|g_{i}(t, 0)\right|\right) d t\right\} \\
\leq \frac{2(L r+a)}{1-\sigma} .
\end{gathered}
$$

Proceeding as above, we obtain that $\left|A_{i}^{2} y(t)\right| \leq L r+a$, $\forall y \in B_{r}$, for $t \in\left(s_{i}, t_{i+1}\right], i \geq 1$. 
Then, for all $x, y \in B_{r}$, we have that

$$
\begin{aligned}
\| A x & +B y \|_{\mathrm{PC}} \\
\leq & \frac{T^{q}(2-\sigma)}{\Gamma(q+1)(1-\sigma)}\left\|m_{f}\right\|_{\mathrm{PC}} h_{f}[r(1+T D)+T K] \\
& +\frac{2(L r+a)}{1-\sigma} \leq r .
\end{aligned}
$$

Step 2. We show that $A^{2}=\sum_{i=1}^{N} A_{i}^{2}$ is a contraction mapping.

From the definition of $A^{2} u(t), A_{i}^{2} u(t)$, and $\left(H_{3}\right)$, we can easily get

$\left|A_{i}^{2} x(t)-A_{i}^{2} y(t)\right| \leq \frac{2 L}{1-\sigma}\|x-y\|_{\mathrm{PC}}, \quad \forall x, y \in B_{r}, \forall t \in J$,

which implies that $A^{2}$ is a contraction mapping.

Step 3. Next we will prove that $A^{1}$ is compact and continuous.

We also divide the proof into 3 steps.

(I) We show that $A^{1}$ is continuous.

Let $\left\{x_{n}\right\}$ be a sequence such that $x_{n} \rightarrow x$ in $\operatorname{PC}(J, R)$. Then for all $t \in J$, by the definition of $A^{1} u(t), A_{i}^{1} u(t)$, we have

$$
\begin{aligned}
&\left|A^{1} x_{n}(t)-A^{1} x(t)\right| \\
& \leq \frac{T^{q}(2-\sigma)}{\Gamma(q+1)(1-\sigma)} \\
& \cdot\left|f\left(t, x_{n}(t), B x_{n}(t)\right)-f(t, x(t), B x(t))\right| \\
& \leq \frac{T^{q}(2-\sigma)}{\Gamma(q+1)(1-\sigma)} \\
& \cdot\left(\left|f\left(t, x_{n}(t), B x_{n}(t)\right)-f\left(t, x(t), B x_{n}(t)\right)\right|\right. \\
&\left.\quad+\left|f\left(t, x(t), B x_{n}(t)\right)-f(t, x(t), B x(t))\right|\right) .
\end{aligned}
$$

From $\left(\mathrm{H}_{2}\right),\left(\mathrm{H}_{4}\right)$, we can get the continuity of $f$ and $B$. Then one has

$$
\left\|A^{1} x_{n}-A^{1} x\right\|_{\mathrm{PC}} \longrightarrow 0, \quad \text { as } x_{n} \longrightarrow x(n \longrightarrow \infty),
$$

which shows that the operator $A^{1}$ is continuous.

(II) We show that $A^{1}$ maps bounded sets into bounded sets in $\mathrm{PC}(J, R)$.

Indeed, it is enough to show that, for any $R>0$, there exists a $R^{\prime}>0$ such that, for each $x \in B_{R}=\{u \in \mathrm{PC}(J, R)$ : $\left.\|u\|_{\mathrm{PC}} \leq R\right\}$, we have $\left\|A^{1} x\right\|_{\mathrm{PC}} \leq R^{\prime}$.

For all $t \in J$, from the definition of $A^{1} u(t), A_{i}^{1} u(t)$, and $\left(H_{2}\right),\left(H_{4}\right)$, one can obtain

$$
\begin{aligned}
& \left|A^{1} x(t)\right| \\
& \quad \leq \frac{T^{q}(2-\sigma)}{\Gamma(q+1)(1-\sigma)}\left\|m_{f}\right\|_{\mathrm{PC}} h_{f}[R(1+T D)+T K]:=R^{\prime} .
\end{aligned}
$$

Then we conclude that $A^{1}$ maps bounded sets into bounded sets in $\operatorname{PC}(J, R)$.

(III) At last, we prove that $A^{1}$ maps bounded sets into equicontinuous sets in $\operatorname{PC}(J, R)$.

For interval $t \in\left(s_{i}, t_{i+1}\right], s_{i} \leq l_{1}<l_{2} \leq t_{i+1}, i=1, \ldots, N$, $\forall x(t) \in B_{r}$, by definition of $A^{1} u(t)$ and $\left(H_{3}\right)$, we have

$$
\begin{aligned}
& \left|\left(A^{1} x\right)\left(l_{2}\right)-\left(A^{1} x\right)\left(l_{1}\right)\right| \\
& =\mid \frac{1}{\Gamma(q)} \int_{s_{i}}^{l_{2}}\left(l_{2}-s\right)^{q-1} f(s, x(s), B x(s)) d s \\
& \quad-\frac{1}{\Gamma(q)} \int_{s_{i}}^{l_{1}}\left(l_{1}-s\right)^{q-1} f(s, x(s), B x(s)) d s \mid \\
& \leq \frac{1}{\Gamma(q)} \int_{l_{1}}^{l_{2}}\left(l_{2}-s\right)^{q-1}|f(s, x(s), B x(s))| d s \\
& \quad+\frac{1}{\Gamma(q)} \int_{s_{i}}^{l_{1}}|f(s, x(s), B x(s))| \\
& \leq \frac{\left\|m_{f}\right\|_{\mathrm{PC}} h_{f}[r(1+T D)+T K]}{\Gamma(q)} \cdot\left[\left(l_{1}-s\right)^{q-1}-\left(l_{2}-s\right)^{q-1}\right] d s \\
& \quad \frac{\left\|m_{f}\right\|_{\mathrm{PC}} h_{f}[r(1+T D)+T K]}{\Gamma(q+1)} \\
& \quad\left\{\int_{l_{1}}^{l_{2}}\left(l_{2}-s\right)^{q-1} d s+\int_{s_{i}}^{l_{1}}\left[\left(l_{1}-s\right)^{q-1}-\left(l_{2}-s\right)^{q-1}\right] d s\right\}
\end{aligned}
$$

which is independent of $x$. As $l_{1} \rightarrow l_{2}$, the right-hand side of the above inequality tends to zero. Therefore $A^{1}$ is equicontinuous on interval $\left(s_{i}, t_{i+1}\right], i \geq 1$.

Proceeding as above, we can also prove that $A^{1}$ is equicontinuous for the time interval $\left[0, t_{1}\right]$. From the definition of $A_{i}^{1}$, it is easy to see that $A^{1}$ is equicontinuous for the other cases.

By Arzela-Ascoli Theorem, $A^{1}$ is continuous and compact.

As a consequence of Lemma 5, we deduce that the operator $A$ has at least a fixed point on $B_{r}$ which means that problem (3) has at least a mild solution.

\section{Examples}

Consider the following impulsive system of fractional differential equations.

Example 1. Consider

$$
\begin{array}{r}
{ }^{c} D_{t}^{1 / 2} u(t)=\frac{1}{16}\left(\frac{u(t) \sin t^{2}}{1+e^{t^{2}}}+\int_{0}^{t} \frac{\sin \left(t^{2}+\sqrt{s}\right)}{2} u(s) d s\right), \\
t \in\left(s_{i}, t_{i+1}\right], \quad i=1, \ldots, N,
\end{array}
$$




$$
\begin{gathered}
u(t)=\frac{u(t)}{18 e^{t}(1+|u(t)|)}, \quad t \in\left(t_{i}, s_{i}\right], i=1, \ldots, N, \\
u(0)=\int_{0}^{1} \frac{u(t)}{3} d t,
\end{gathered}
$$

where $0=t_{0}=s_{0}<t_{1}=1 / 2 \leq s_{1} \leq \cdots \leq t_{N} \leq s_{N} \leq t_{N+1}=1$ are pre-fixed numbers, $J=[0,1], q=1 / 2$, and $w(t)=1 / 3<$ 1 .

We prove that Example 1 satisfies all the assumptions of Theorem 7.

In Example 1, set

$$
\begin{gathered}
f(t, u, v)=\frac{u \sin t^{2}}{16\left(1+e^{t^{2}}\right)}+\frac{v}{16}, \\
B u(t)=\int_{0}^{t} \frac{\sin \left(t^{2}+\sqrt{s}\right)}{2} u(s) d s, \\
k(t, s, u)=\frac{\sin \left(t^{2}+\sqrt{s}\right)}{2} u .
\end{gathered}
$$

It is easy to see that $f$ is jointly continuous. We can also check that $\left(H_{1}\right)$ and $\left(H_{2}\right)$ are satisfied with $e(t)=1 / 16$ and $d(t)=1 / 2$.

For $t \in[0,1], u \in R$, and $g_{i}(t, u)=u / 18 e^{t}(1+|u|)$, then we know

$$
\left|g_{i}(t, u)-g_{i}(t, v)\right| \leq \frac{1}{18}|u-v|,
$$

with $l_{i}(t)=1 / 36$, so $\left(H_{3}\right)$ is also satisfied.

From 1 , we can get $L=1 / 18, T=1, M=1 / 16, D=1 / 2$, $q=1 / 2, \Gamma(3 / 2)=\sqrt{\pi} / 2, \sigma=1 / 6$, and then

$$
n=\frac{T^{q} M(1+T D)(2-\sigma)}{\Gamma(q+1)(1-\sigma)}+\frac{2 L}{1-\sigma}=\frac{33}{80 \sqrt{\pi}}+\frac{2}{15}<1 .
$$

So all the conditions of Theorem 7 are satisfied. As a consequence of Theorem 7, Example 1 has a unique mild solution.

\section{Example 2. Consider}

$$
\begin{aligned}
& { }^{c} D_{t}^{1 / 2} u(t) \\
& =\frac{1}{15\left(1+e^{\sqrt{\sin t}}\right)}\left(u(t)+\int_{0}^{t} \frac{\sin \left(t^{2}+\sqrt{s}\right)}{2} u(s) d s\right), \\
& t \in\left(s_{i}, t_{i+1}\right], \quad i=1, \ldots, N \\
& u(t)=\frac{u(t)}{18(1+|u(t)|)}, \quad t \in\left(t_{i}, s_{i}\right], i=1, \ldots, N, \\
& u(0)=\int_{0}^{1} \frac{u(t)}{2} d t
\end{aligned}
$$

where $0=t_{0}=s_{0}<t_{1}=1 / 3 \leq s_{1} \leq t_{2} \leq \cdots \leq t_{N} \leq$ $s_{N} \leq t_{N+1}=1$ are prefixed numbers, $J=[0,1], q=1 / 2$, and $w(t)=1 / 2<1$.
It is easy to see that $g_{i}(t, 0)=0$ is bounded. Set

$$
\begin{gathered}
f(t, u, v)=\frac{u+v}{15\left(1+e^{\sqrt{\sin t}}\right)}, \\
B u(t)=\int_{0}^{t} \frac{\sin \left(t^{2}+\sqrt{s}\right)}{2} u(s) d s, \\
k(t, s, u)=\frac{\sin \left(t^{2}+\sqrt{s}\right)}{2} u .
\end{gathered}
$$

Then we have

$$
|f(t, u, B u)| \leq \frac{1}{15}|u+B u|
$$

with $m_{f}=1 / 15 \in C\left(J, R^{+}\right), h_{f}(|u+B u|)=|u+B u| \epsilon$ $C\left(R^{+}, R^{+}\right)$being nondecreasing. So $\left(H_{4}\right)$ is satisfied. Similarly to the proof of Example 1, we know that $\left(H_{2}\right)$ and $\left(H_{3}\right)$ are satisfied.

From (51), we can obtain $T=1, \sigma=1 / 6, D=1 / 2, L=$ $1 / 18<1, a=0, K=0, \Gamma(3 / 2)=\sqrt{\pi} / 2, m_{f}=1 / 15, q=1 / 2$, and $2 L /(1-\sigma)=2 / 15<1$, and then the inequality (32) becomes $13 r / 18 \geq(11 / 30 \sqrt{\pi}) r$. Hence, inequality (32) holds for all $r>0$.

Thus, all the assumptions in Theorem 8 are satisfied, and our results can be applied to Example 2. So Example 2 has at least one mild solution.

\section{Conflict of Interests}

The authors declare that there is no conflict of interests regarding the publication of this paper.

\section{Acknowledgments}

This work is supported by National Natural Science Foundation of China (nos. 11001274, 11101126, and 11261010), China Postdoctoral Science Foundation (no. 20110491249), Key Scientific and Technological Research Project of Department of Education of Henan Province (no. 12B110006), Youth Science Foundation of Henan University of Science and Technology (no. 2012QN010), and Innovative Natural Science Foundation of Henan University of Science and Technology (no. 2013ZCX020). The authors thank the referees for their careful reading of the paper and insightful comments, which help to improve the quality of the paper. The authors would also like to acknowledge the valuable comments and suggestions from the editors, which vastly contribute to the perfection of the paper.

\section{References}

[1] A. M. Samoilenko and N. A. Perestyuk, Impulsive Differential Equations, vol. 14 of World Scientific Series on Nonlinear Science, Series A: Monographs and Treatises, World Scientific Publishing, Singapore, 1995.

[2] V. Lakshmikantham, D. D. Bainov, and P. S. Simeonov, Theory of Impulsive DifferentialEquations, vol. 6 of Series in Modern Applied Mathematics, World Scientific, Singapore, 1989. 
[3] M. Benchohra, J. Henderson, and S. Ntouyas, Impulsive Differential Equations and Inclusions, vol. 2 of Contemporary Mathematics and Its Applications, Hindawi Publishing Corporation, New York, NY, USA, 2006.

[4] M. Frigon and D. O’Regan, "Existence results for first-order impulsive differential equations," Journal of Mathematical Analysis and Applications, vol. 193, no. 1, pp. 96-113, 1995.

[5] S. M. Afonso, E. M. Bonotto, M. Federson, and S. Schwabik, "Discontinuous local semiflows for Kurzweil equations leading to LaSalle's invariance principle for differential systems with impulses at variable times," Journal of Differential Equations, vol. 250, no. 7, pp. 2969-3001, 2011.

[6] S. M. Afonso, E. M. Bonotto, M. Federson, and L. P. Gimenes, "Boundedness of solutions of retarded functional differential equations with variable impulses via generalized ordinary differential equations," Mathematische Nachrichten, vol. 285, no. 5-6, pp. 545-561, 2012.

[7] S. M. Afonso, E. M. Bonotto, M. Federson, and L. P. Gimenes, "Stability of functional differential equations with variable impulsive perturbations via generalized ordinary differential equations," Bulletin des Sciences Mathématiques, vol. 137, no. 2, pp. 189-214, 2013.

[8] E. Hernández and D. O’Regan, “On a new class of abstract impulsive differential equations," Proceedings of the American Mathematical Society, vol. 141, no. 5, pp. 1641-1649, 2013.

[9] M. Pierri, D. O’Regan, and V. Rolnik, "Existence of solutions for semi-linear abstract differential equations with not instantaneous impulses," Applied Mathematics and Computation, vol. 219, no. 12, pp. 6743-6749, 2013.

[10] Y. Zhou and F. Jiao, "Nonlocal Cauchy problem for fractional evolution equations," Nonlinear Analysis. Real World Applications, vol. 11, no. 5, pp. 4465-4475, 2010.

[11] Y. Zhou, F. Jiao, and J. Li, "Existence and uniqueness for fractional neutral differential equations with infinite delay," Nonlinear Analysis: Theory, Methods \& Applications, vol. 71, no. 7-8, pp. 3249-3256, 2009.

[12] K. Diethelm, The Analysis of Fractional Differential Equations, vol. 2004 of Lecture Notes in Mathematics, Springer, 2010.

[13] A. A. Kilbas, M. H. Srivastava, and J. J. Trujillo, "Theory and applications of fractional differential equations," in NorthHolland Mathematics Studies, vol. 204, Elsevier Science B.V., Amsterdam, The Netherlands, 2006.

[14] V. Lakshmikantham, S. Leela, and J. Vasundhara Devi, Theory of Fractional Dynamic Systems, Cambridge Scientific Publishers, Cambridge, UK, 2009.

[15] K. S. Miller and B. Ross, An Introduction to the Fractional Calculus and Fractional Differential Equations, John Wiley \& Sons, New York, NY, USA, 1993.

[16] I. Podlubny, Fractional Differential Equations, Academic Press, San Diego, Calif, USA, 1999.

[17] V. E. Tarasov, Fractional Dynamics: Application of Fractional Calculus to Dynamics of Particles, Fields and Media, Nonlinear Physical Science, Springer, Heidelberg, Germany, 2011.

[18] R. P. Agarwal, M. Benchohra, and S. Hamani, "A survey on existence results for boundary value problems of nonlinear fractional differential equations and inclusions," Acta Applicandae Mathematicae, vol. 109, no. 3, pp. 973-1033, 2010.

[19] M. Benchohra, J. Henderson, S. K. Ntouyas, and A. Ouahab, "Existence results for fractional order functional differential equations with infinite delay," Journal of Mathematical Analysis and Applications, vol. 338, no. 2, pp. 1340-1350, 2008.
[20] J. Wang and Y. Zhou, "A class of fractional evolution equations and optimal controls," Nonlinear Analysis: Real World Applications, vol. 12, no. 1, pp. 262-272, 2011.

[21] J. Wang, Y. Zhou, and W. Wei, "A class of fractional delay nonlinear integrodifferential controlled systems in Banach spaces," Communications in Nonlinear Science and Numerical Simulation, vol. 16, no. 10, pp. 4049-4059, 2011.

[22] S. Zhang, "Existence of positive solution for some class of nonlinear fractional differential equations," Journal of Mathematical Analysis and Applications, vol. 278, no. 1, pp. 136-148, 2003.

[23] J. M. Gallardo, "Second-order differential operators with integral boundary conditions and generation of analytic semigroups," The Rocky Mountain Journal of Mathematics, vol. 30, no. 4, pp. 1265-1291, 2000.

[24] G. L. Karakostas and P. C. Tsamatos, "Multiple positive solutions of some Fredholm integral equations arisen from nonlocal boundary value problems," Electronic Journal of Differential Equations, vol. 30, pp. 1-17, 2002.

[25] A. Lomtatidze and L. Malaguti, "On a nonlocal boundary value problems for second order nonlinear singular differential equations," Georgian Mathematical Journal, vol. 7, no. 1, pp. 133$154,2000$.

[26] C. Corduneanu, Integral Equations and Applications, Cambridge University Press, Cambridge, UK, 1991.

[27] R. P. Agarwal and D. O’Regan, Infinite Interval Problems for Differential, Difference and Integral Equations, Kluwer Academic, Dordrecht, The Netherlands, 2001.

[28] B. Ahmad and A. Alsaedi, "Existence of approximate solutions of the forced Duffing equation with discontinuous type integral boundary conditions," Nonlinear Analysis. Real World Applications, vol. 10, no. 1, pp. 358-367, 2009.

[29] A. Boucherif, "Second-order boundary value problems with integral boundary conditions," Nonlinear Analysis, vol. 70, no. 1, pp. 364-371, 2009.

[30] Z. Yang, "Existence of nontrivial solutions for a nonlinear Sturm-Liouville problem with integral boundary conditions," Nonlinear Analysis: Theory, Methods \& Applications, vol. 68, no. 1, pp. 216-225, 2008.

[31] X. Zhang, M. Feng, and W. Ge, "Existence results for nonlinear boundary-value problems with integral boundary conditions in Banach spaces," Nonlinear Analysis: Theory, Methods \& Applications, vol. 69, no. 10, pp. 3310-3321, 2008.

[32] A. Anguraj, P. Karthikeyan, M. Rivero, and J. J. Trujillo, "On new existence results for fractional integro-differential equations with impulsive and integral conditions," Computers \& Mathematics with Applications, vol. 66, no. 12, pp. 2587-2594, 2014.

[33] M. A. Krasnoselskii, Topological Methods in the Theory of Nonlinear Integral Equations, Pergamon Press, New York, NY, USA, 1964. 


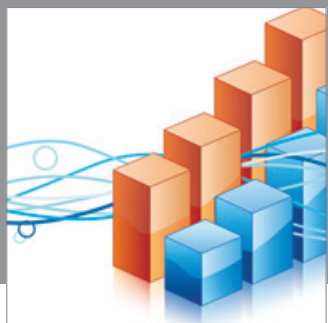

Advances in

Operations Research

mansans

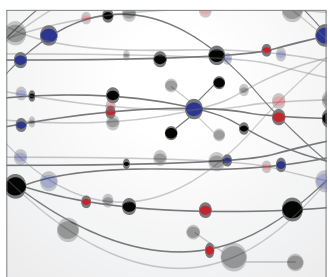

The Scientific World Journal
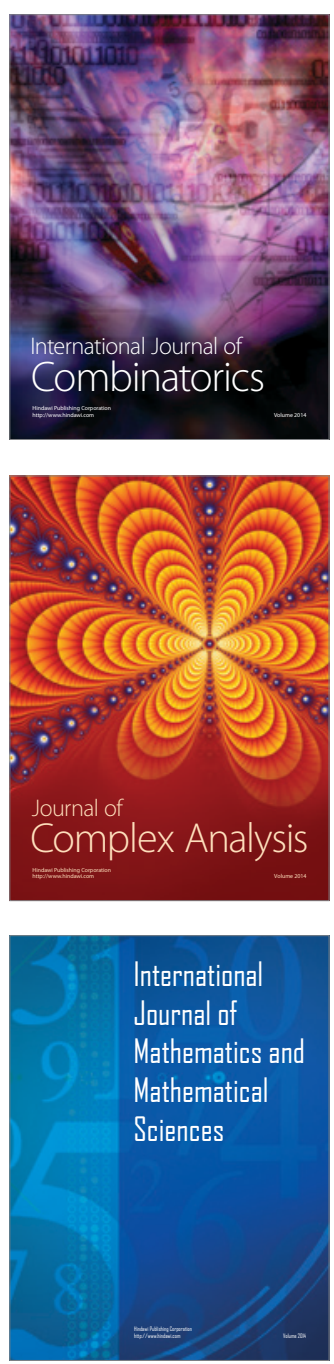
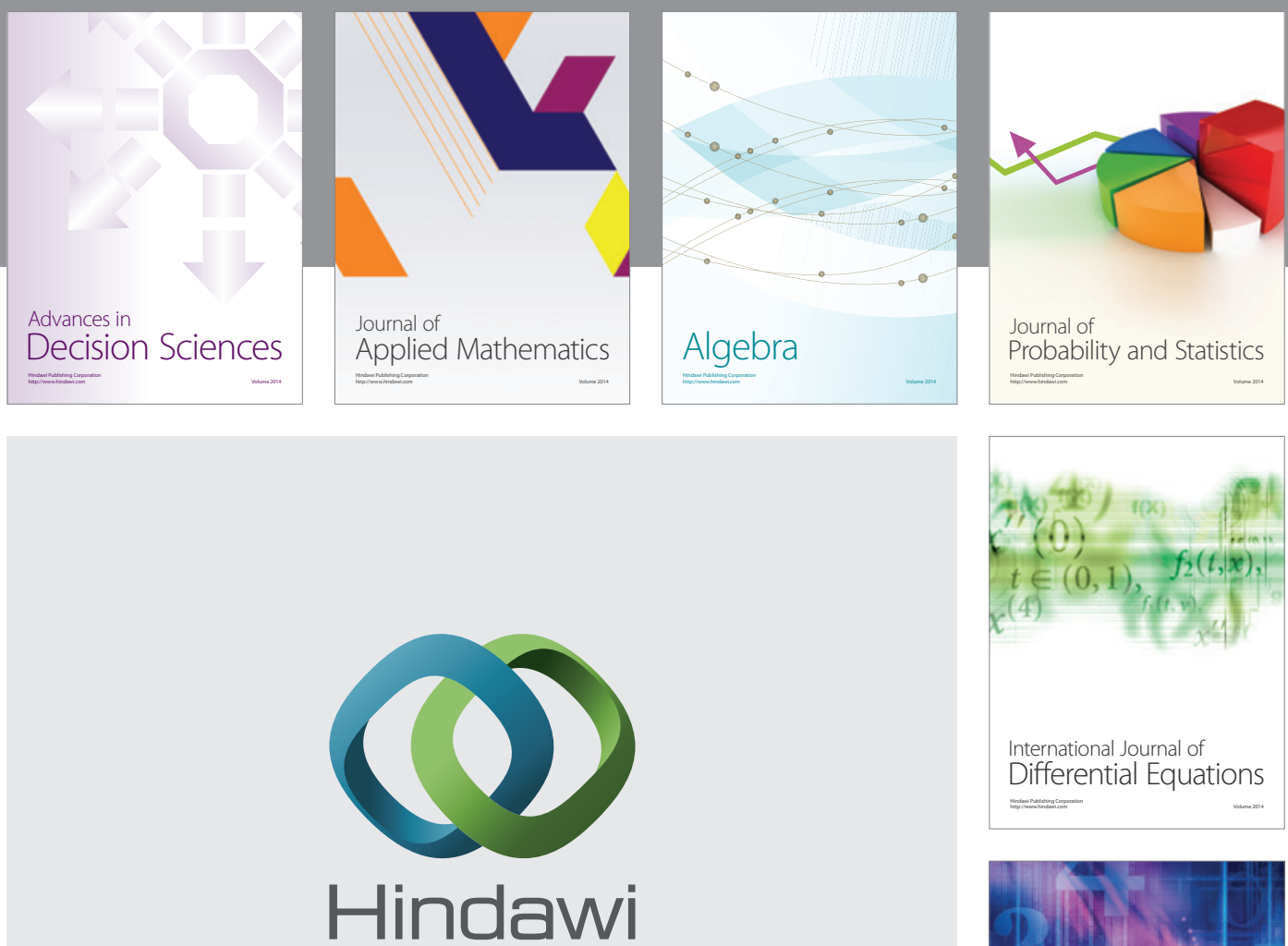

Submit your manuscripts at http://www.hindawi.com
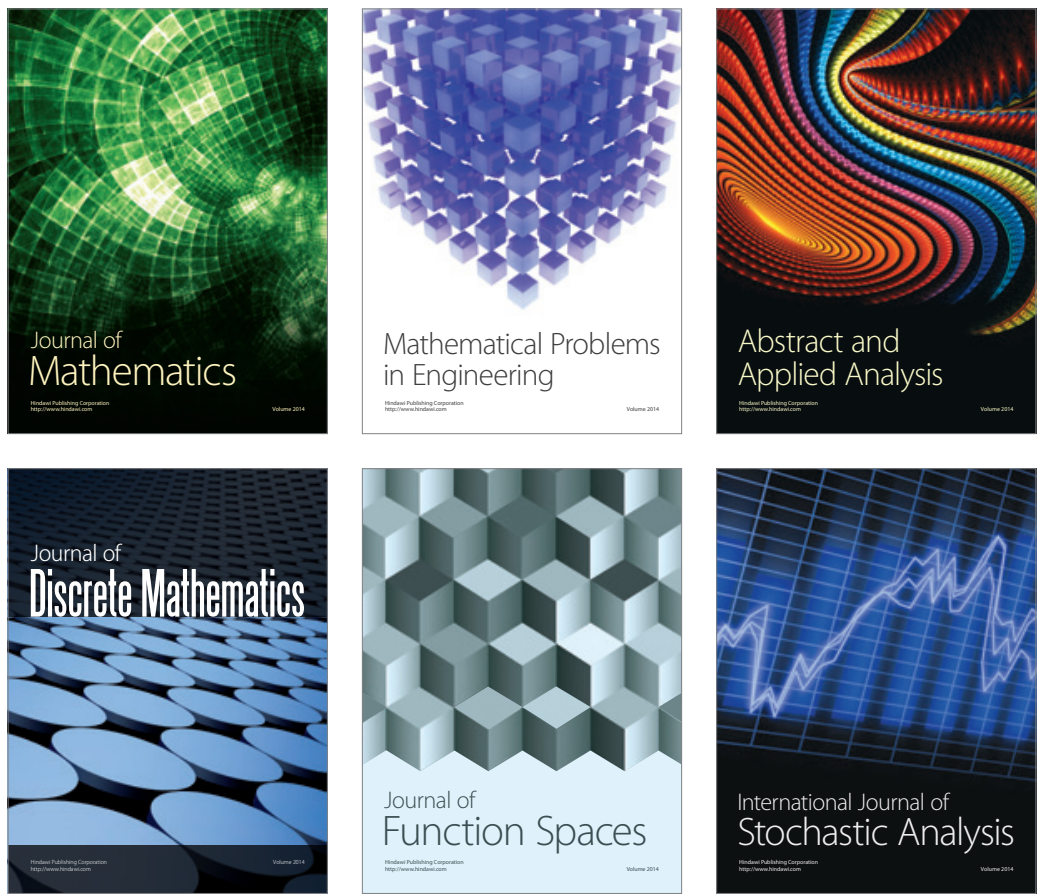

Journal of

Function Spaces

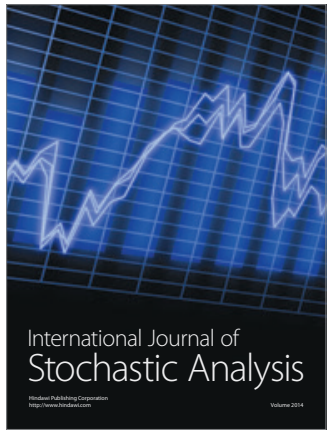

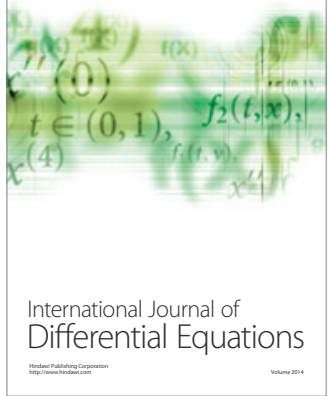
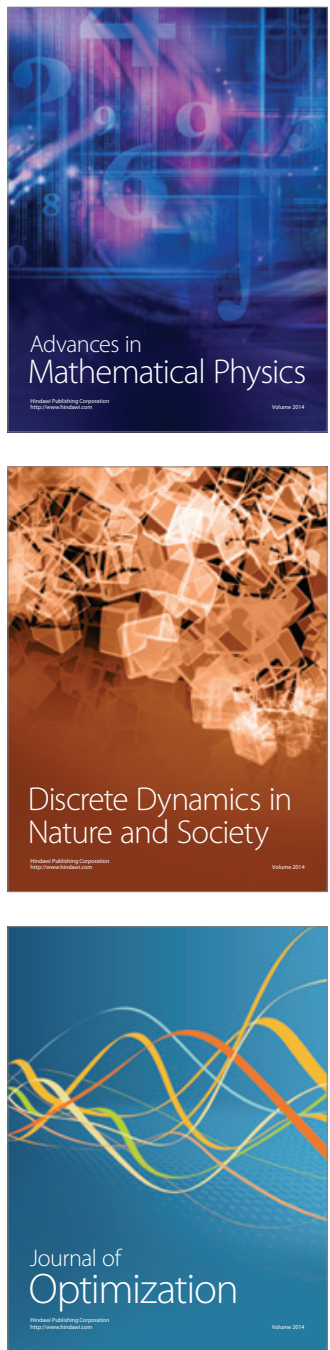